\title{
Dielectric properties of SPC/E and TIP4P under the static electric field and microwave field
}

\author{
Di Li and Guo-zhu Jia* \\ College of physical and Electronics Engineering, Sichuan Normal University, \\ Chengdu, China
}

\begin{abstract}
Nonequilibrium molecular dynamics simulations of the SPC/E and TIP4P models have been performed both in the absence and presence of the static electric field $\left(0-3 \times 10^{9} \mathrm{~V} / \mathrm{m}\right)$ and the microwave field $\left(2.45 \mathrm{G}, 0-3 \times 10^{9} \mathrm{~V} / \mathrm{m}\right)$. The radial distribution function, dielectric constant, polarizability, dielectric relaxation time, hydrogen bonding and diffusion coefficient were investigated. Significant alteration is the static electric field has effect on the tetrahedral structure of water; the interaction between microwave and water depends on the hydrogen bonding. The $3 \times 10^{7} \mathrm{~V} / \mathrm{m}$ is threshold intensity, as the intensity of the static electric field increases the decline of the dielectric constant becomes more pronounced, and the polarizability obviously increased with the increase of microwave field. And the life of hydrogen bonding is oscillatory under the high static electric field strength.
\end{abstract}

Keywords: SPC/E; TIP4P; microwave; dielectric properties; molecular dynamics

\section{Introduction}

Water exists as a kind of widely used solvents in the earth, the properties of water play an irreplaceable role in biology, chemistry, physics and other fields [1-3]. There have been many experimental methods and theoretical studies on the water, including the far-infrared [4], X-ray absorption spectroscopy [5], the NMR Spectroscopy [1], the dielectric relaxation spectroscopy (DRS) [6], IR spectroscopy [7] and molecular dynamics (MD) simulations [8-10]. Comparing to these methods, the MD simulations

*Corresponding author. Email: jia1689500@126.com 
could better trace the detail of motion path, show relatively accurate estimation of water properties [11], it also expose the structure and some changes at the microscopic level [12] and show molecular motions on the picoseconds time scale [13]. What calls for special attention is correctly selecting the water models, because they are not only meaningful for the MD simulations but also important for the accurate calculation of the potential parameters [14]. Besides, the dielectric constant represents a very good test for the accuracy of an inter-molecular potential [6], there are many researches which are about the dielectric constant of water models, including SPC [12], TIP5P [15], TIP4P/2005 [14], SPC/E [16], SPC/E and SWM4-DP [9], TIP4P/2005, TIP4P/2005f and TTM3F [17], TIP4P $\mathrm{FQ}_{\mathrm{FQ}}[3,18,19]$ and $\mathrm{SPC}_{\mathrm{FW}}$ etc [3].

In recent years, microwave has been widely applied on the organic chemistry [20, 21], biology [22], physio-chemical researches [23-28], industrial and agricultural production [29]. So, studying the dielectric properties of water models with the microwave field becomes more meaningful work for microwave energy application. Although Niall J. English [27] has carried out an investigation about the influence that the microwave filed caused on a rigid/polarizable and a flexible/nonpolarizable potential model, at present, less survey has involved the comparison between the water models with the high strength filed yet. Therefore, in this paper, the intention was extended to track the influence on the dielectric properties of SPC/E and TIP4P, including the radial distribution function, dielectric constant, polarizability, dielectric relaxation time, hydrogen bonding and diffusion coefficient, which were caused by the different intensities of the static electric field and the microwave filed.

\section{Computational Method}

\subsection{Interaction potentials and simulation details}

The simulations were performed using a modified version of the GROMACS 4.5.5[30] simulation package and GROMOS [31] force fields. The simulations involved $512 \mathrm{SPC} / \mathrm{E}$ and TIP4P were respectively placed in a cubic simulation periodic box and two concentrations, static electric field and microwave field. Water 
molecules were added to the system from a relaxed liquid configuration at $298 \mathrm{~K}$ and 1 bar.

Prior to system relaxation MD, energy minimization was carried out with a composite protocol of steepest descent, conjugate gradient, and truncated Newton steps, using termination gradients of 5000, 1000, and $100 \mathrm{~kJ} / \mathrm{mol} \cdot \mathrm{nm}$, respectively. MD is coupled to a modified Nose'-Hoover thermostat [32] system. The Particle Mesh-Ewald method [33, 34] was used to compute long-range electrostatics to within a relative tolerance of $1 \times 10^{-6}$. A cut-off distance of $12.5 \AA$ was applied to real-space Ewald interactions. The same value was used for Van der Waals interactions. The LINCS algorithm [35] was applied to constrain bond lengths to hydrogen atoms. A leap-frog algorithm [36] was used with a time step of 1 fs.

\subsection{Incorporation of static electric filed and microwave field}

To investigate the influence of the external static electric field and the microwave field on the SPC/E and the TIP4P, a series of intensities of the static electric fields and the microwave fields were applied to the water models with frequency $2.45 \mathrm{GHz}$. The microwave field was represented by a homogeneous time-alternating electric field of the form:

$$
E_{\text {ext }}(t)=E_{0} \cos (\omega t) x_{0}, B=0
$$

Prior to production simulations a $1 \mathrm{~ns}$ NVT relaxation run and a $1 \mathrm{~ns}$ NPT relaxation run were performed. Then equilibrium MD simulations were performed in the NPT ensemble and the fist $3 \mathrm{~ns}$ were considered as further relaxation, keeping the rest $7 \mathrm{~ns}$ simulation time as production run. Then a series of NNPT simulations were carried out for $10 \mathrm{~ns}$ at $298 \mathrm{~K}$ in fields of frequency $2.45 \mathrm{GHz}$ with RMS intensities of $0,3 \times 10^{5} \mathrm{~V} / \mathrm{m}, 3 \times 10^{6} \mathrm{~V} / \mathrm{m}, 3 \times 10^{7} \mathrm{~V} / \mathrm{m}, 3 \times 10^{8} \mathrm{~V} / \mathrm{m}$ and $3 \times 10^{9} \mathrm{~V} / \mathrm{m}$, using the relaxed system as a starting configuration. Simulation details refer to [37].

The dielectric constants were calculated based on the fluctuation in total dipole moment $M=\sum_{i} \mu i$ by:

$$
\varepsilon_{s}=1+\frac{4 \pi}{3 V k_{B} T \varepsilon_{0}}\left(\left\langle M^{2}\right\rangle-\langle M\rangle^{2}\right)
$$


Where $\varepsilon_{0}$ is the vacuum permittivity, $V$ is the volume, $K_{B}$ is Boltzmann's constant and $T$ is the temperature.

The Debye relaxation time constant can be calculated from non-equilibrium MD simulations when an external field is applied [38]. The dipole moment along the field's direction $\langle M\rangle$ can be related to the Debye relaxation time by Eq.(3) [38]:

$$
\tau=\tau_{s} \frac{\left[\varepsilon_{0}+2+C_{\mathrm{rf}}\left(\varepsilon_{0}-1\right)\right]}{3}
$$

The parameter $C_{\mathrm{rf}}$ can be calculated by Eq.(4) [38]:

$$
C_{\mathrm{rf}}=\frac{\left(2 \varepsilon_{0}-2 \varepsilon_{R F}\right)\left(1+k R_{c}\right)-\varepsilon_{R F}\left(k R_{c}\right)^{2}}{\left(\varepsilon_{0}+2 \varepsilon_{R F}\right)\left(1+k R_{c}\right)+\varepsilon_{R F}\left(k R_{c}\right)^{2}}
$$

where $k$ is the Debye screening length, $R_{c}$ is the cut-off radius, and $\tau$ is the Debye relaxation time.

The relaxation time $\tau_{s}$ can be obtained from the average dipole moment upon

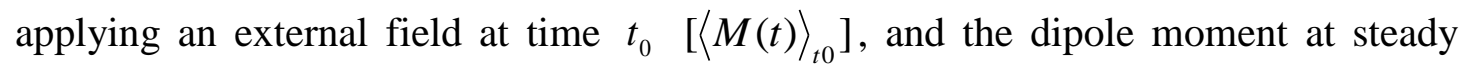
state $\left[\langle M(t=\infty)\rangle_{t}\right]$, by Eq.(5)[38]:

$$
\langle M(t)\rangle_{t 0}=\langle M(t=\infty)\rangle_{t}\left[1-\exp \left(-\frac{t-t_{0}}{t_{s}}\right)\right]
$$

The molecular polarzabilities were calculated by:

$$
\varepsilon_{s}-1=4 \pi \frac{p}{\varepsilon_{0} E}
$$

Where $p$ is the molecular polarizability and $E$ is the intensity of the external field.

\section{Results and discussion}

\subsection{The radial distribution function}

Here we focused on a smaller subset of radial distribution function (RDF) to detect the dielectric constant changes of the SPC/E and TIP4P under the high static electric field and microwave field.

The two fields caused the influence on the potential of two water models are 
mainly presented by the RDF of $g_{H-O}, g_{O-O}$ and $g_{H-H}$ (Fig.1 and Fig.2). At the fig. $1\left(\mathrm{a}_{1}, \mathrm{a}_{2}\right.$ and $\left.\mathrm{a}_{3}\right)$ and fig.2( $\mathrm{a}_{1}, \mathrm{a}_{2}$ and $\left.\mathrm{a}_{3}\right)$, the heights of the first peak of $g_{H-O}$, $g_{O-O}$ and $g_{H-H}$ are in each set of profiles declines as the intensity of the static electric field increases. This indicates that the distribution of molecules becomes more regular when a high-strength uniform static electric fled is applied. However, at the fig. $1\left(a_{4}, a_{5}\right.$ and $\left.a_{6}\right)$ and fig.2( $a_{4}, a_{5}$ and $\left.a_{6}\right)$, the overlapping of the curve is perfect from 0 to $3 \times 10^{9} \mathrm{~V} / \mathrm{m}$, the peak heights of the $g_{H-O}, g_{O-O}$ and $g_{H-H}$ almost don't shift, this phenomenon illustrates that the strength of the microwave field doesn't act on the first peak heights of the $g_{H-O}, g_{O-O}$ and $g_{H-H}$ for the SPC/E and TIP4P models.
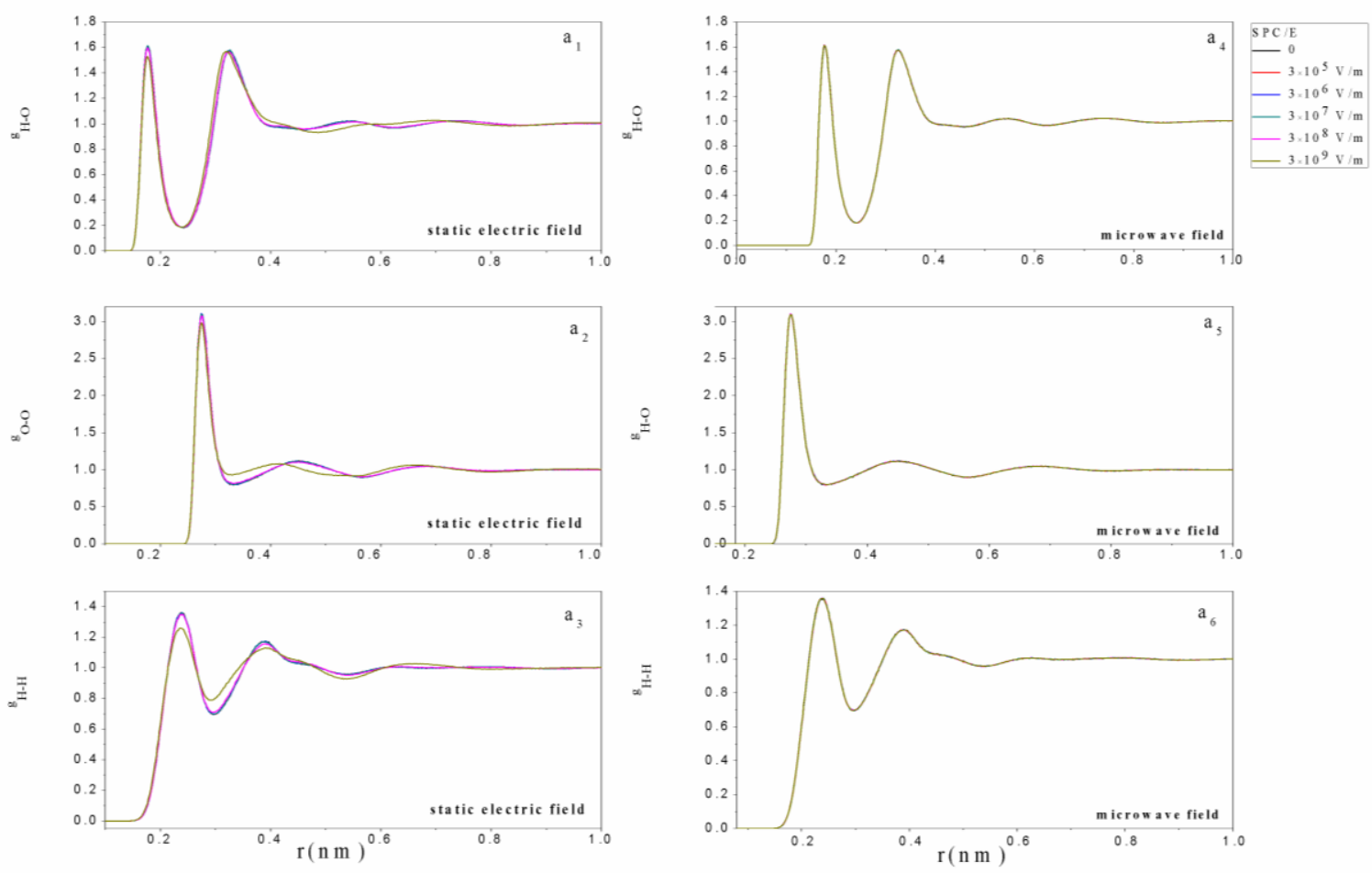

Fig. 1. The RDF of SPC/E is in the absence and presence of the static electric field and the microwave field 

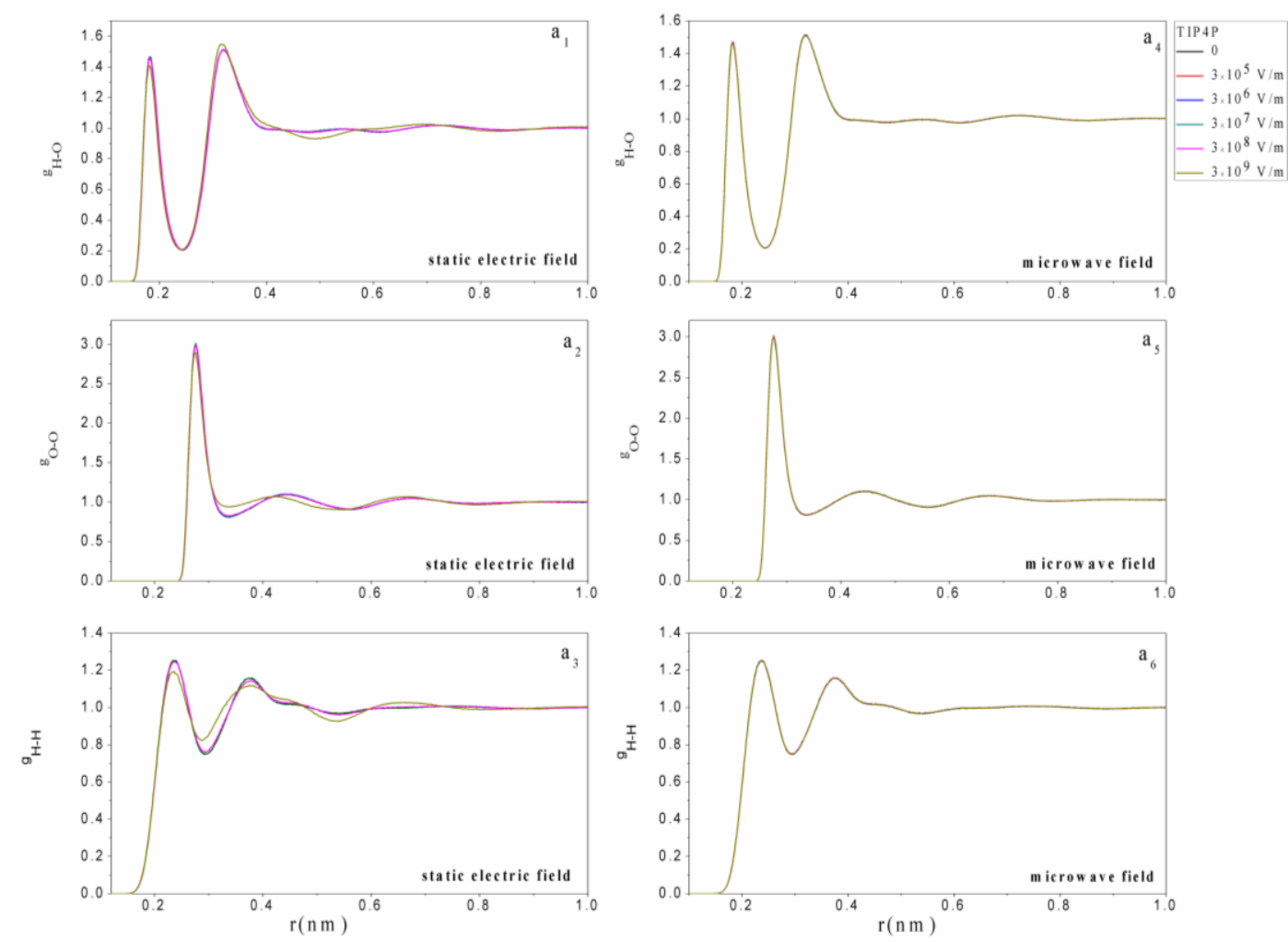

Fig. 2. The RDF of TIP4P is in the absence and presence of the static electric field and the microwave field
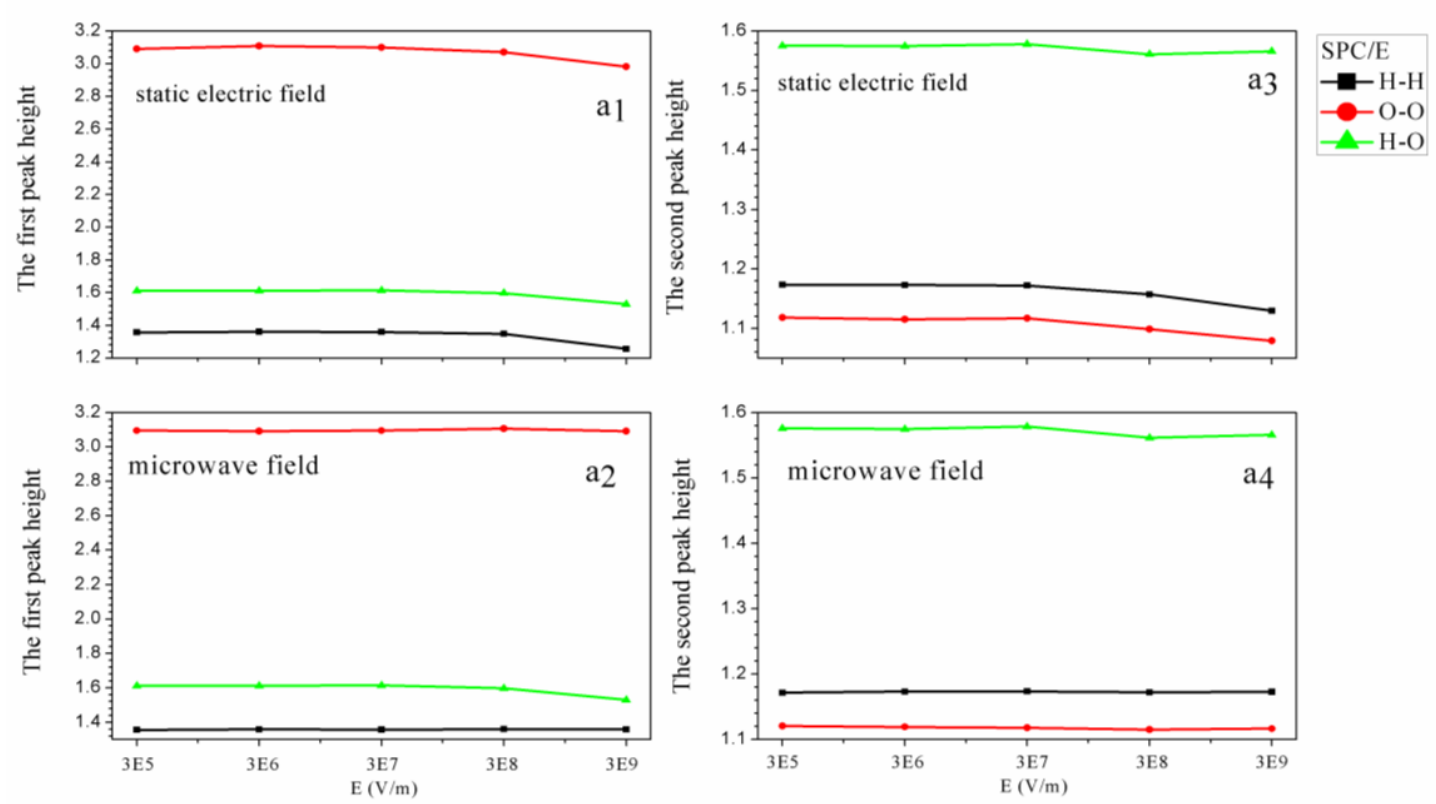

Fig. 3. The first peak heights and the second peak heights of SPC/E are in the absence and presence of the static electric field and the microwave field 

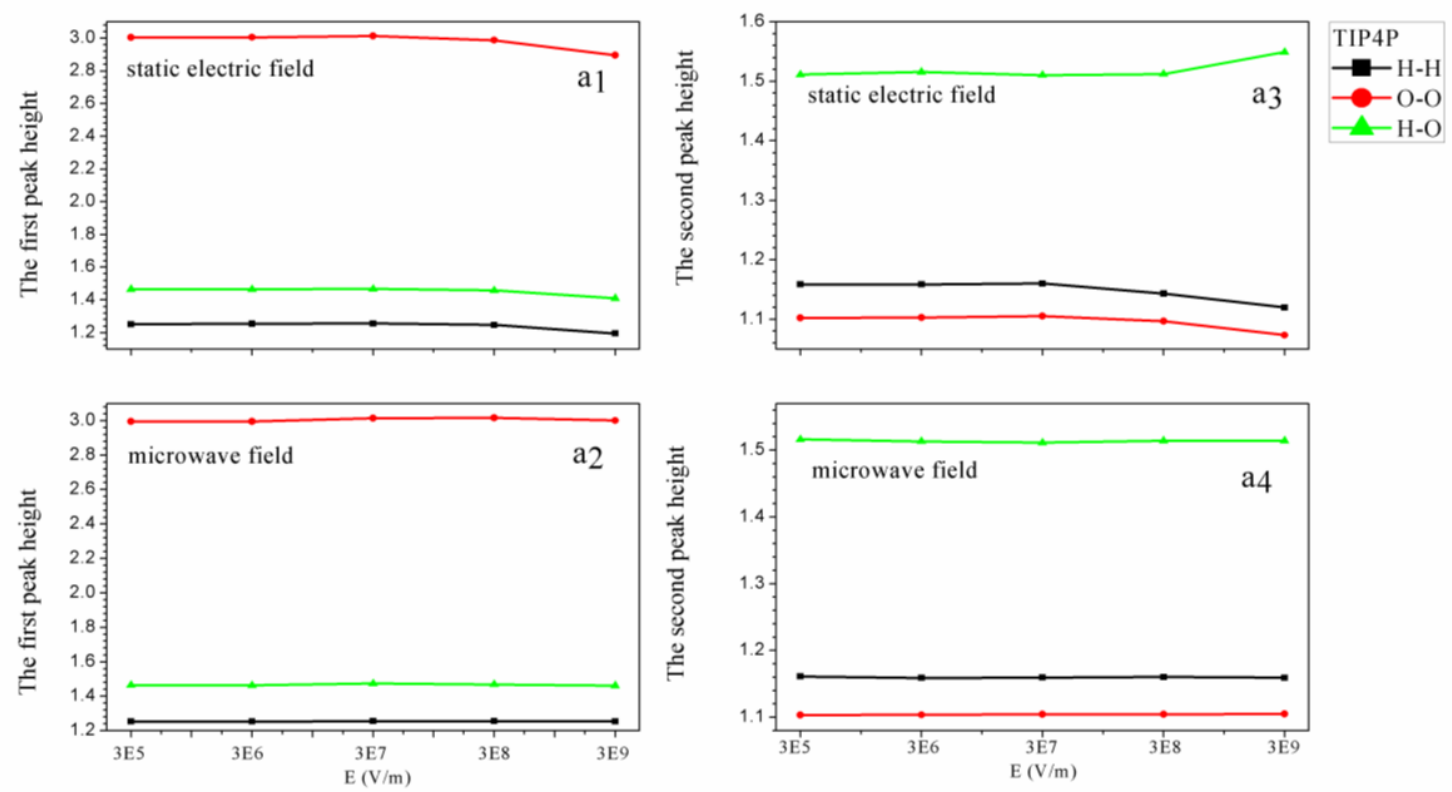

Fig. 4. The first peak heights and the second peak heights of TIP4P are in the absence and presence of the static electric field and the microwave field

To further analyze the changes of the peak heights, we combined the first and second peaks of the SPC/E and TIP4P with the two fields in the fig. 3 and fig.4. At the fig. 3 ( $a_{1}$ and $a_{3}$ ), the first and second peaks both present a reduced tendency at high strength field with the static electric field. At the fig. 3 ( $a_{2}$ and $a_{4}$ ) the first and second peak heights of the $g_{O-O}$ and $g_{H-H}$ aren't affected by the microwave field. However, the peak heights of $g_{H-O}$ expresses a tiny reduced tendency with the high-strength microwave field. For the TIP4P model, the first and second peak heights both decline at the high-strength field with the static electric field, the first peak height of the $g_{H-O}$ will reduce at the high field, but the second peak height of the $g_{H-O}$ increases when the field strength is high. However, the microwave field doesn't affect the first and second peak heights. Arguably, the static electric field acts on the tetrahedral structure of water. And the hydrogen bonding plays a main role in the interaction between the microwave and water.

\subsection{The dielectric constant}

Figs.5 and 6 exposed the variation of the dielectric constant with the static electric 
field and the microwave field whose strengths are from 0 to $3 \times 10^{9} \mathrm{~V} / \mathrm{m}$. The dielectric constant of SPC/E and TIP4P are agreement with the experimental data [8] when the field strength is 0 .
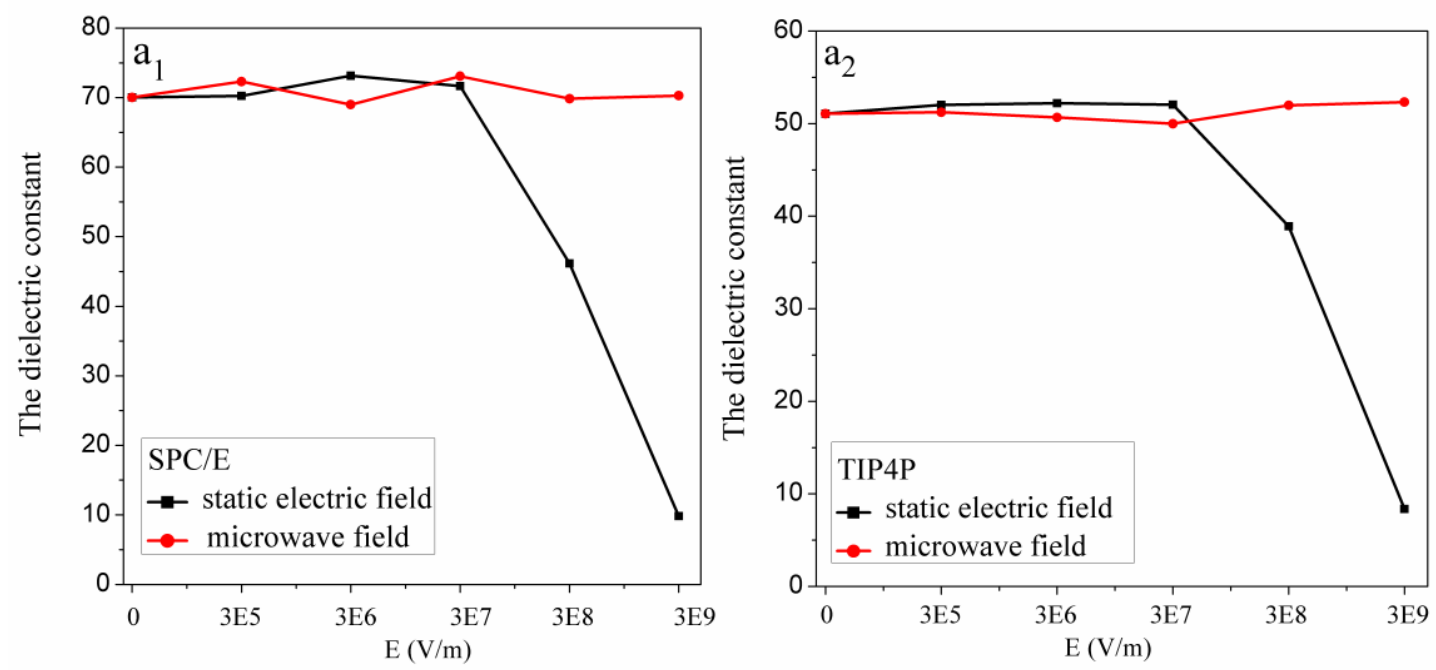

Fig. 5. The dielectric constant of SPC/E and TIP4P in the absence and presence of the static electric field and the microwave field

The relationship between the dielectric constant and the applied two kinds of external field, which are static electric field and microwave field, is given by the formula (2).

The dielectric constants of the SPC/E and the TIP4P at $298 \mathrm{~K}$ with different intensities of static electric field and microwave field are obtained in fig.5 $\left(a_{1}, a_{2}\right)$. Above $3 \times 10^{7} \mathrm{~V} / \mathrm{m}$, the dielectric constants of the SPC/E and TIP4P start to obviously reduce under the static dielectric field. There are tiny variations with the microwave field. The numbers of the electric constant are different every time, but it will be stable in an interval. In short, the dielectric constant doesn't change clearly as the strength of the microwave field increases. The changes still aren't significant with the microwave field.

\subsection{The polarisability}



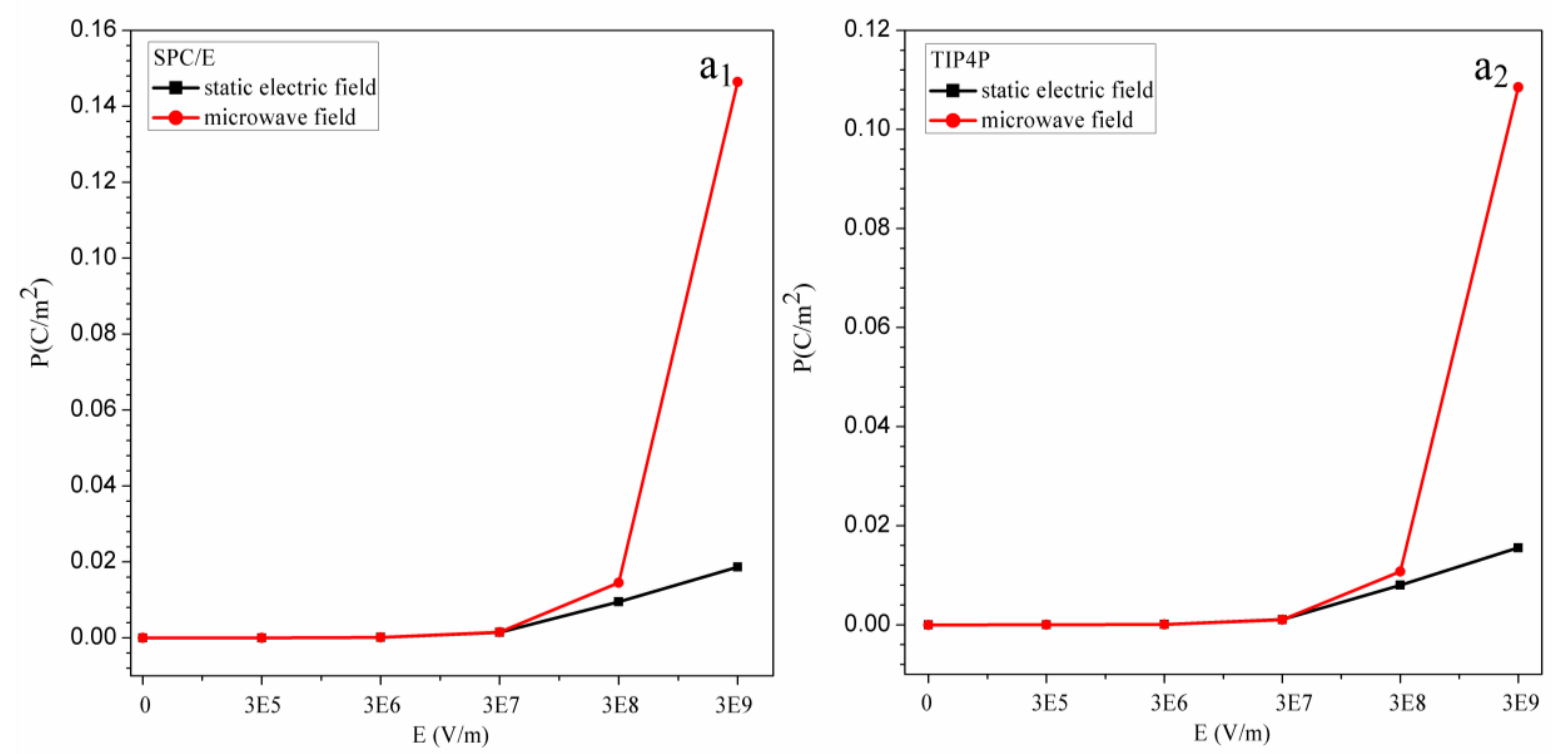

Fig. 6. The polarizability of the SPC/E and TIP4P in the absence and presence of the static electric field and the microwave field

Fig.6 $\left(a_{1}, a_{2}\right)$ illustrates the variation of the molecular polarzability of the two kinds of water models as a function of the static electric field or the microwave field. As the fig.6 $\left(a_{1}\right)$ reflects that there is an enhancement above $3 \times 10^{7} \mathrm{~V} / \mathrm{m}$ under the microwave field, however, as the static electric field was applied, it has no significant effect on the polarzability. There are the same results for the TIP4P at the fig.6( $\left.\mathrm{a}_{2}\right)$. The influences are all bigger when apply the microwave than that when the static electric field is applied. This indicates the influences of the microwave field on the polarzability of two water models are more obvious than that with the static electric field. And it also shows that the direction of the molecular dipole moment is gradually with the same direction of the microwave field.

\subsection{The relaxation time constant}

The accuracy of the estimated relaxation time constant varied depending on the applied electric field strength. This may be because dissimilar field strengths perturb the molecules in various ways, causing the molecular dipoles to return to their equilibrium state differently [39].

Fig. $7\left(a_{1}, a_{2}\right)$ shows that the variation of dielectric relaxation time is as a function 
of the static electric field and the microwave field. As shown in the fig.7( $\left.a_{1}\right)$, it is found that the dielectric relaxation time shows a tendency which is first increased and then decrease as the static electric field strength increases. As for the microwave field, the relaxation time showns a first decreased and then increased tendency. At the fig. $7\left(\mathrm{a}_{2}\right)$, the variation of dielectric relaxation time is stable before $3 \times 10^{8} \mathrm{v} / \mathrm{m}$, above this, it remarkably increases with the static electric field. With the microwave field, the variation shows an irregular pattern. All changes due to that the water molecules attempt to reorient more rapidly in response to the fields, however, at higher frequencies and strength field the dipole alignment becomes very weak and the relaxation time becomes negligible. The relaxation time declines as a function of field strength while the system's dipole response is still appreciable, as the greater magnitude of the rotational force exerted on molecules accelerates the speed at which molecules reorient. Although, the relaxation times all exposed some changes with the two fields, we can still find that the microwave filed caused poorer changes comparing the static electric field.
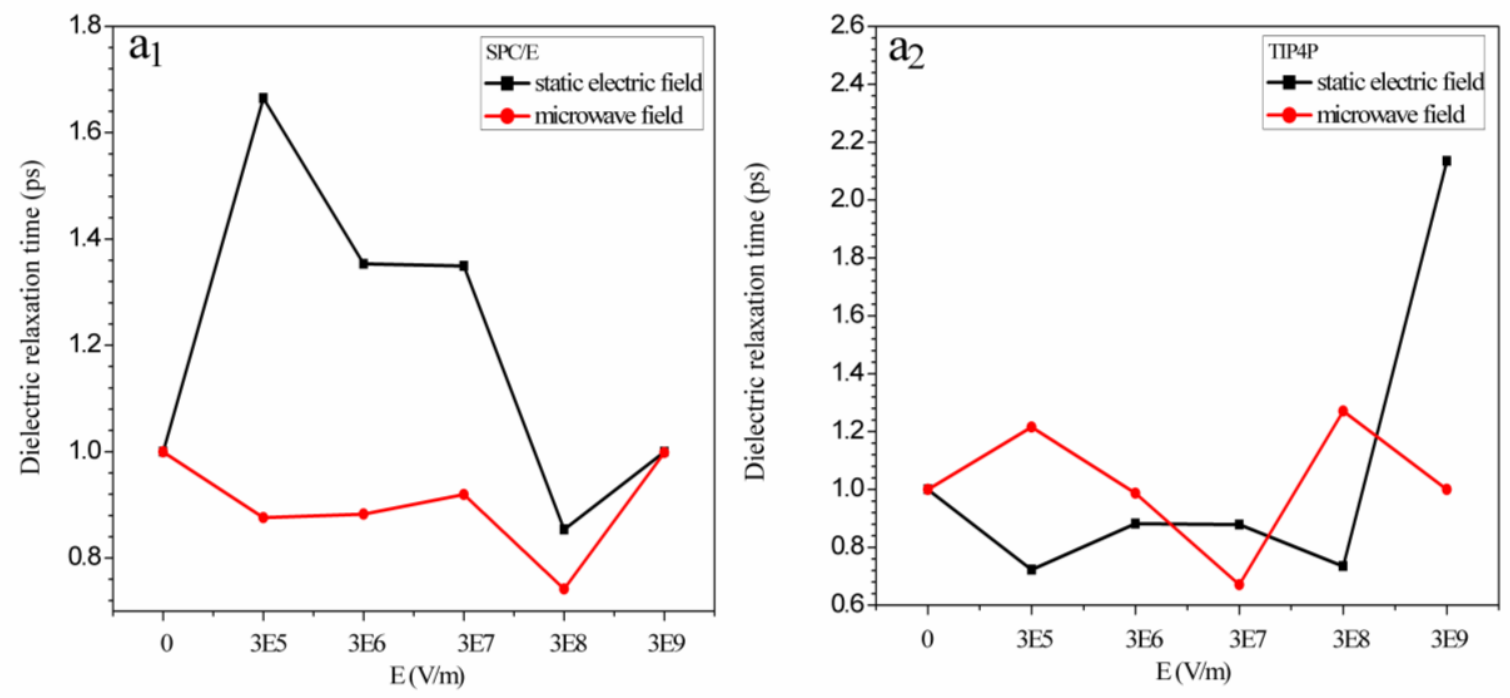

Fig. 7. Variation in dielectric relaxation time of SPC/E and TIP4P as a function of the static electric field and the microwave field

\subsection{The hydrogen bonding}



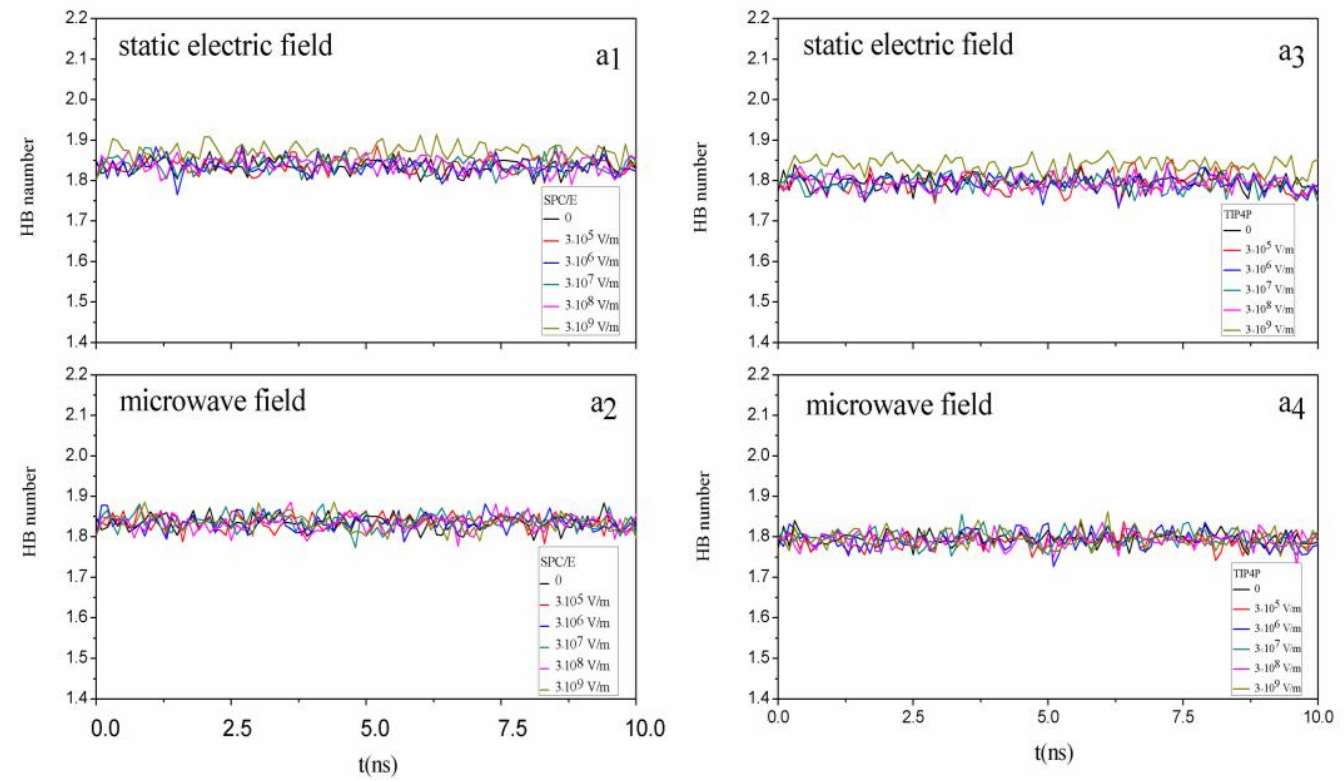

Fig. 8. The hydrogen bonding number of SPC/E and TIP4P for the different strength of the electric filed and the microwave field

From the fig.8, it can be assessed that the applications of the static electric field and the microwave field have a major influence on the average hydrogen-bond number of the SPC/E and the TIP4P. It is found that for the average hydrogen-bond numbers of the SPC/E and the TIP4P exhibits a stable number. The result shows that the two fields both don't affect the hydrogen-bond numbers.
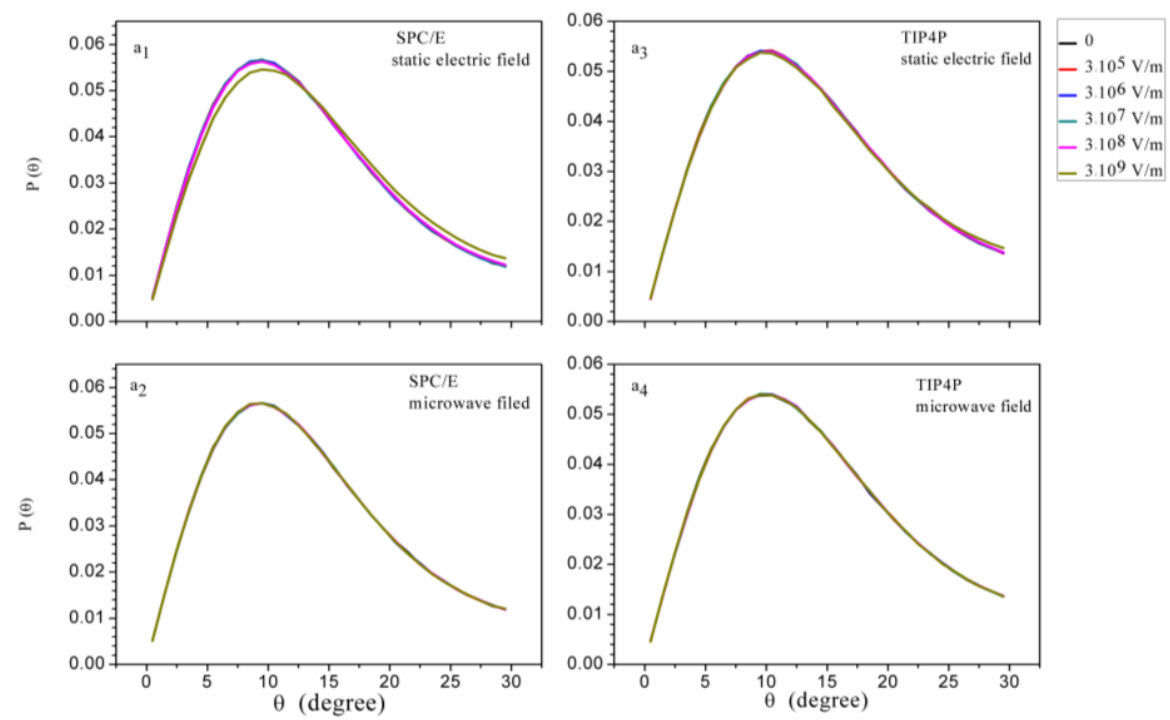

Fig. 9. The bond angle of SPC/E and TIP4P in the absence and presence of the static electric field and the microwave field

Analyzing the fig.9, we know that the biggest degree is about 10. From the picture 
$a_{1}$, the probability of the bond will reduce with the strength of the static electric field increases before the degree is about 12.5. After this, the probability of the bond has an increased trend as the intensity increases. From the fig. $9\left(a_{3}, a_{4}\right)$, the probability shows an unapparent change for the TIP4P. This may be caused by the reassignment of charges because of the action of static electric field. However, the microwave field has no effect on the two water models.
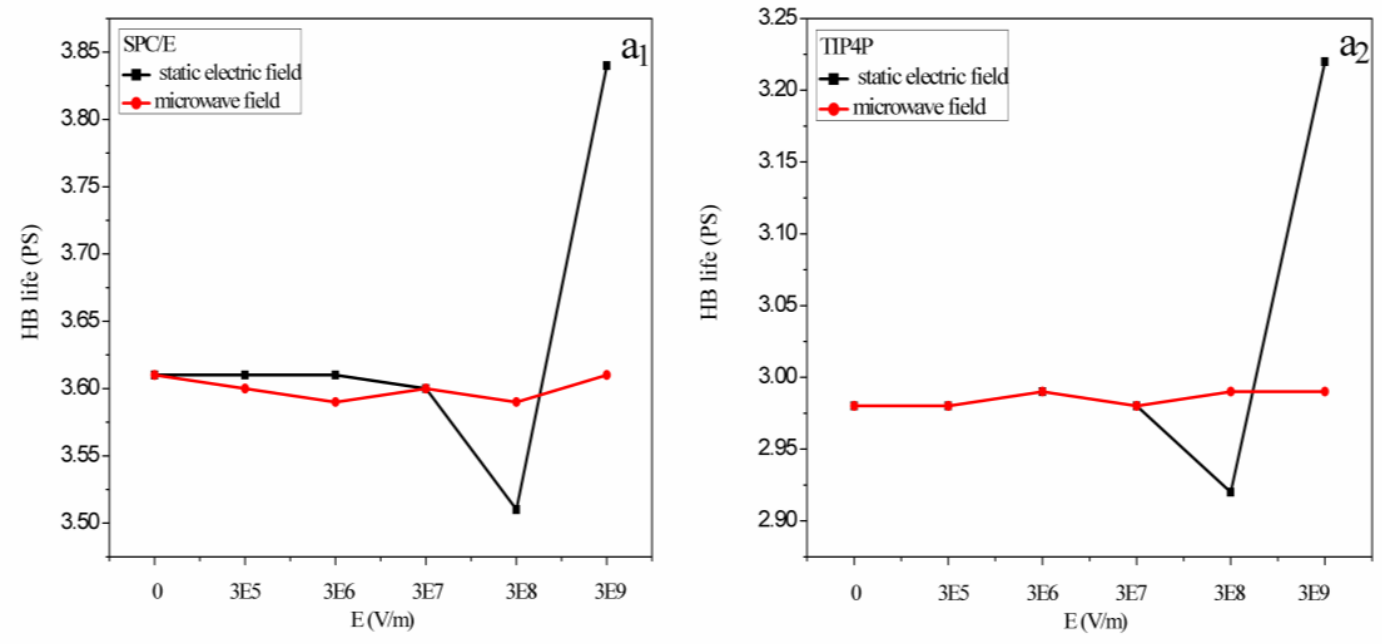

Fig. 10. The life of hydrogen bond of SPC/E and TIP4P in the absence and presence of the static electric field and the microwave field

We estimate the hydration-bond lifetime, the fig.10 $\left(a_{1}, a_{2}\right)$ have depicted relevant information. In general, the hydrogen bond lifetimes are more stable for the SPC/E and TIP4P models with the microwave field. Arguably, there is an oscillatory motion above $3 \times 10^{7} \mathrm{~V} / \mathrm{m}$ with high static electric field. The result is almost consistent for the two models. This illustrates that the field intensity of microwave field doesn't affect hydrogen bond lifetimes, and the static electric field will cause some changes, and the field strength is more high, the changes more clear.

\subsection{The diffusion coefficient}



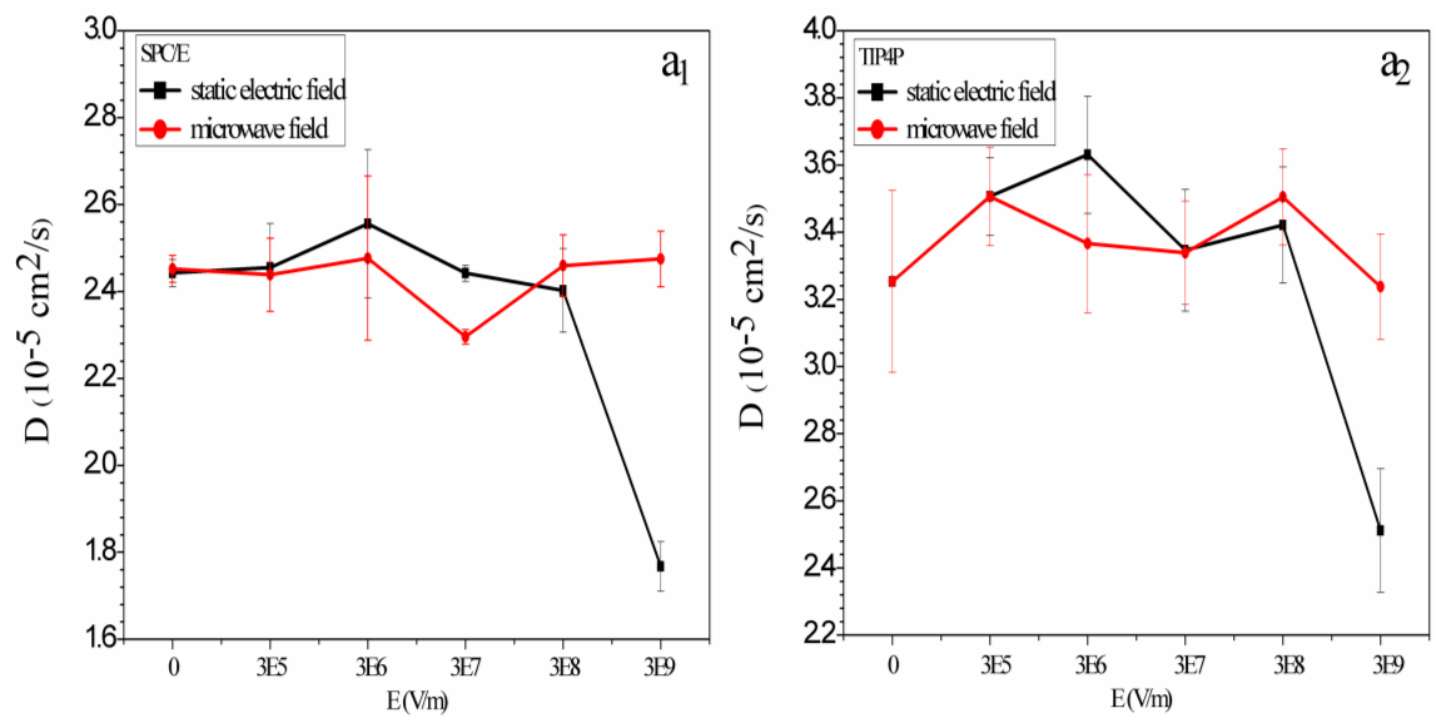

Fig. 11. The diffusion coefficient of SPC/E and TIP4P in the absence and presence of the static electric field and the microwave field

The diffusion coefficient is an important property of water models. The fig.11( $\mathrm{a}_{1}$, $a_{2}$ ) shows the diffusion coefficient of the two water models, fig.11( $\left.a_{1}\right)$ exposes that the diffusion coefficient of the SPC/E will have a small rise from $3 \times 10^{5} \mathrm{~V} / \mathrm{m}$ to $3 \times 10^{6}$ $\mathrm{V} / \mathrm{m}$ under the static electric field, after that, the diffusion coefficients start to reduce. As for the microwave field, there is an oscillatory change which starts from $3 \times 10^{5} \mathrm{~V} / \mathrm{m}$, but the change isn't noticeable, and it also doesn't present disciplinarian oscillatory. Of course, there are errors exist, and we found the big error exits at $3 \times 10^{6} \mathrm{~V} / \mathrm{m}$ both under the static electric field and the microwave field. It is found that the errors are roughly the same. For the TIP4P, we observed that the diffusion coefficient keeps minute oscillatory, whereas the oscillatory doesn't has a rule. The same as that the SPC/E the errors are roughly consistent. This situation can be explained that the dipole relaxation time is reduced and the proportional dipole alignment increases as a function of intensity.

\section{Conclusions}

The first peak, the second peak and the dielectric constant will reduce with the field strength increases under high static electric field, it exposes that the static electric field has contributed on the tetrahedral structure. The hydrogen bonding 
occupies primary position and the polarizability is also meaningful in the interaction between microwave and water. For the relaxation time, the microwave field caused lesser changes comparing to the static electric field. Moreover, the alterations of the life of hydrogen bonding and the diffusion coefficient are more obvious as the static electric fields are applied. The numbers of the hydrogen bonding aren't influenced by the static electric field and the microwave field, and the similar behavior is find for bond angle. In contrast to the static electric field, the microwave field didn't cause pronounced influence on the dielectric constant, dielectric relaxation time, hydrogen bonging and diffusion coefficient of the SPC/E and TIP4P, even the field strength had reached to $3 \times 10^{9} \mathrm{~V} / \mathrm{m}$, this reveals the SPC/E and TIP4P can be used to describe the interaction between microwave and water within a short simulation time with molecular dynamic simulation, and can simulate interaction between the microwave and aqueous solution.

\section{Acknowledgements}

This work was supported by the National Science Foundation of China [grant number 61102044].

\section{References}

[1] H. Yoo, R. Paranji, G.H. Pollack, Impact of hydrophilic surfaces on interfacial water dynamics probed with NMR spectroscopy, The journal of physical chemistry letters, 2 (2011) 532-536.

[2] G. Raabe, R.J. Sadus, Molecular dynamics simulation of the dielectric constant of water: The effect of bond flexibility, The Journal of chemical physics, 134 (2011) 234501.

[3] Y. Wu, H.L. Tepper, G.A. Voth, Flexible simple point-charge water model with improved liquid-state properties, The Journal of chemical physics, 124 (2006) 024503.

[4] N.J. English, J. MacElroy, Hydrogen bonding and molecular mobility in liquid water in external electromagnetic fields, The Journal of chemical physics, 119 (2003) 11806-11813.

[5] J.-J. Velasco-Velez, C.H. Wu, T.A. Pascal, L.F. Wan, J. Guo, D. Prendergast, M. Salmeron, The structure of interfacial water on gold electrodes studied by x-ray absorption spectroscopy, Science, 346 (2014) 831-834.

[6] Z. Lu, E. Manias, D.D. Macdonald, M. Lanagan, Dielectric relaxation in dimethyl sulfoxide/water mixtures studied by microwave dielectric relaxation spectroscopy, The Journal of Physical Chemistry A, 113 (2009) 12207-12214. 
[7] T. Shimamori, A. Fujii, Infrared Spectroscopy of Warm and Neutral Phenol-Water Clusters, The Journal of Physical Chemistry A, 119 (2015) 1315-1322.

[8] D. van der Spoel, P.J. van Maaren, H.J. Berendsen, A systematic study of water models for molecular simulation: derivation of water models optimized for use with a reaction field, The Journal of chemical physics, 108 (1998) 10220-10230.

[9] O. Gereben, L. Pusztai, On the accurate calculation of the dielectric constant from molecular dynamics simulations: The case of SPC/E and SWM4-DP water, Chemical Physics Letters, 507 (2011) 80-83.

[10] I.-C. Yeh, M.L. Berkowitz, Dielectric constant of water at high electric fields: molecular dynamics study, The Journal of chemical physics, 110 (1999) 7935-7942.

[11] H. Berendsen, J. Grigera, T. Straatsma, The missing term in effective pair potentials, Journal of Physical Chemistry, 91 (1987) 6269-6271.

[12] E. Guardia, J. Martí, L. García-Tarrés, D. Laria, A molecular dynamics simulation study of hydrogen bonding in aqueous ionic solutions, Journal of Molecular Liquids, 117 (2005) 63-67.

[13] A.Y. Zasetsky, I.M. Svishchev, Dielectric response of concentrated $\mathrm{NaCl}$ aqueous solutions: Molecular dynamics simulations, The Journal of chemical physics, 115 (2001) 1448-1454.

[14] J.L. Abascal, C. Vega, A general purpose model for the condensed phases of water: TIP4P/2005, The Journal of chemical physics, 123 (2005) 234505.

[15] S.W. Rick, A reoptimization of the five-site water potential (TIP5P) for use with Ewald sums, The Journal of chemical physics, 120 (2004) 6085-6093.

[16] M.R. Reddy, M. Berkowitz, The dielectric constant of SPC/E water, Chemical Physics Letters, 155 (1989) 173-176.

[17] D.C. Elton, M.-V. Fernández-Serra, Polar nanoregions in water: A study of the dielectric properties of TIP4P/2005, TIP4P/2005f and TTM3F, The Journal of chemical physics, 140 (2014) 124504.

[18] N.J. English*, Molecular dynamics simulations of liquid water using various long-range electrostatics techniques, Molecular Physics, 103 (2005) 1945-1960.

[19] S.W. Rick, S.J. Stuart, B.J. Berne, Dynamical fluctuating charge force fields: Application to liquid water, The Journal of chemical physics, 101 (1994) 6141-6156.

[20] Q. Jie, J. Guo-Zhu, Dielectric Constant of Polyhydric Alcohol-DMSO Mixture Solution at the Microwave Frequency, The Journal of Physical Chemistry A, 117 (2013) 12983-12989.

[21] J.-c. Liu, G.-Z. Jia, F.-h. Liu, Dielectric properties of pyridine N-oxide aqueous solution under the static electric field and microwave field, Molecular Physics, (2015) 1-11.

[22] K. Grenier, D. Dubuc, P.-E. Poleni, M. Kumemura, H. Toshiyoshi, T. Fujii, H. Fujita, Resonant based microwave biosensor for biological cells discrimination, in: Radio and Wireless Symposium (RWS), 2010 IEEE, IEEE, 2010, pp. 523-526.

[23] N. English, Molecular dynamics simulations of microwave effects on water using different long-range electrostatics methodologies, Molecular Physics, 104 (2006) 243-253.

[24] G. Roussy, J.A. Pearce, Foundations and industrial applications of microwave and radio frequency fields: physical and chemical processes, John Wiley \& Sons Inc, 1995.

[25] M. Tanaka, M. Sato, Microwave heating of water, ice, and saline solution: Molecular dynamics study, The Journal of chemical physics, 126 (2007) 034509.

[26] Q. Liu, F. Weng, S.J. English, An improved fast microwave water emissivity model, Geoscience and Remote Sensing, IEEE Transactions on, 49 (2011) 1238-1250.

[27] N.J. English, J. MacElroy, Molecular dynamics simulations of microwave heating of water, The 
Journal of chemical physics, 118 (2003) 1589-1592.

[28] C. Gabriel, S. Gabriel, E.H. Grant, B.S. Halstead, Dielectric parameters relevant to microwave dielectric heating, Chemical Society Reviews, 27 (1998) 213-224.

[29] J.-C. Liu, G.-Z. Jia, S. Liu, Dielectric relaxation in isobutyl alcohol-NMP and isobutyl alcohol-DMSO mixtures studied by microwave dielectric relaxation spectroscopy, Colloid and Polymer Science, 293 (2015) 289-296.

[30] B. Hess, C. Kutzner, D. Van Der Spoel, E. Lindahl, GROMACS 4: algorithms for highly efficient, load-balanced, and scalable molecular simulation, Journal of chemical theory and computation, 4 (2008) 435-447.

[31] N. Schmid, C.D. Christ, M. Christen, A.P. Eichenberger, W.F. van Gunsteren, Architecture, implementation and parallelisation of the GROMOS software for biomolecular simulation, Computer Physics Communications, 183 (2012) 890-903.

[32] D.J. Evans, B.L. Holian, The nose-hoover thermostat, The Journal of chemical physics, 83 (1985) 4069-4074.

[33] U. Essmann, L. Perera, M.L. Berkowitz, T. Darden, H. Lee, L.G. Pedersen, A smooth particle mesh Ewald method, The Journal of chemical physics, 103 (1995) 8577-8593.

[34] L.G. Astrakas, C. Gousias, M. Tzaphlidou, Structural destabilization of chignolin under the influence of oscillating electric fields, Journal of Applied Physics, 111 (2012) 074702.

[35] B. Hess, P-LINCS: A parallel linear constraint solver for molecular simulation, Journal of chemical theory and computation, 4 (2008) 116-122.

[36] W. Van Gunsteren, H. Berendsen, A leap-frog algorithm for stochastic dynamics, Molecular Simulation, 1 (1988) 173-185.

[37] N.J. English, G.Y. Solomentsev, P. O’Brien, Nonequilibrium molecular dynamics study of electric and low-frequency microwave fields on hen egg white lysozyme, The Journal of chemical physics, 131 (2009) 035106.

[38] S. Riniker, A.-P.E. Kunz, W.F. van Gunsteren, On the calculation of the dielectric permittivity and relaxation of molecular models in the liquid phase, Journal of chemical theory and computation, 7 (2011) 1469-1475.

[39] C.D. Abeyrathne, M.N. Halgamuge, P.M. Farrell, E. Skafidas, Dielectric properties of liquid phase molecular clusters using the external field method: molecular dynamics study, Physical Chemistry Chemical Physics, 16 (2014) 13943-13947. 